Original Research Paper

\title{
Enhancing the Robustness of Visual Degradation Based HAM Reversible Data Hiding
}

\author{
${ }^{1}$ Habiba Sultana, ${ }^{2}$ A.H.M. Kamal and ${ }^{3}$ Mohammad Mahfuzul Islam \\ ${ }^{I}$ Department of Computer Science and Engineering, \\ Jatiya Kabi Kazi Nazrul Islam University, Trishal, Mymensing, P.C. 2220, Bangladesh \\ ${ }^{2,3}$ Department of Computer Science and Engineering, \\ Bangladesh University of Engineering and Technology, Dhaka, Bangladesh
}

Article history

Received: 21-01-2015

Revised: $14-03-2016$

Accepted: 26-03-2016

Corresponding Author:

A.H.M. Kamal

Department of Computer

Science and Engineering,

Bangladesh University of

Engineering and Technology,

Dhaka, Bangladesh

Email: kamal@jkkniu.edu.bd

\begin{abstract}
Purposely visual degradation of image quality is a newfangled idea in the area of Histogram Association Mapped (HAM) reversible data hiding. Such scheme first divides the color scale, i.e., 0-255, into some partitions by the range, difference of minimum and maximum value of a block. Next, the histogram of a block resided to a partition, original partition, is moved, e.g., shifted, to another partition named reflective partition. First, an association between the original and reflected partition are defined by a chunk of secret message and then the translations of the bins of the histogram of the original partition to the reflective partition are performed to complete the message concealment. In such schemes, the blocks are classified as Reflective Block (RB) and Non-Reflective Block (NRB) based on whether the range is less than 127 or not. Experimentally, it is observed that most of the RB blocks occupy the portion of two partitions which introduce a dilemma in defining original partition as well as associating and hence translating bins. Again, at NRB the range of occupied bins in the histogram of a block is enough longer than the available bins where to reflect. Therefore, the policy of associations and then translations of bins of histogram may force the pixel values to exceed the extremes of gray scale which is indeed impossible. Solutions to these issues are not presented by any literature so far we have studied. Besides, the side information database that is practiced to assist in decoding at receiver is made longer by storing bundle of information and sometimes it becomes near or more than both the secret data and size of image. Resolutions to these stated observations are presented in this article. Experimental results justify the necessity of mitigating these observations.
\end{abstract}

Keywords: Embedding, Histogram Association, Histogram Mapping, Histogram Partition, Side Information, Wheel-Scale

\section{Introduction}

Image steganography is an art of hiding secret information to a carrier image, called cover image. The image with embedded information is termed as stego image. Image steganography can be classified into two broad groups: Irreversible and reversible based on the capability of the scheme of regenerating the cover image from stego. Among the reversible schemes, Histogram Manipulation (HM) based data hiding methods (Ong et al., 2014; Ni et al., 2006; Tai et al., 2009; Tsai et al., 2009; Hong and Chen, 2010; Hong, 2012; Lu and Huang, 2014; Chen et al., 2013) cover a wider range of research area of image steganography. Generally it can be eloquently ascertained that researchers' aspiration is to minimize the distortion of stego image (Ni et al., 2006; Tai et al., 2009; Tsai et al., 2009; Hong and Chen, 2010; Hong, 2012; Lu and Huang, 2014; Chen et al., 2013)- that carries the hidden message. In the referred scheme (Ni et al., 2006; Tai et al., 2009; Tsai et al., 2009; Hong and Chen, 2010; Hong, 2012; Lu and Huang, 2014; Chen et al., 2013) secret data are concealed to the highest peaks of pixels' histogram (Ni et al., 2006), pixel's differences histogram (Tai et al., 2009) or errors' histogram (Tsai et al., 2009; Hong and Chen, 2010; 
Hong, 2012; Lu and Huang, 2014; Chen et al., 2013). Embedding to errors histogram is accomplished by two steps. Firstly predictions are performed by some prediction rules. Thenceforth prediction errors are measured deducing those predicted values from the cover pixels. Embedding to those errors is a very commonly used domain at RDH. However, the process suffers from less embedding capacity as well as degradation of visual quality. Another effort is devoted in 2014 ( $\mathrm{Lu}$ and Huang, 2014) to increase the embedding capacity by regrouping the referenced pixels as pyramidal structure. Doing so the scheme has stepped-up the embedding capacity as well as the growth factor of distortions too. Applying asymmetric histogram shifting, a new way of reducing the degradation of visual quality, but not demolished, is demonstrated by Chen et al. (2013). Again, a double time data embedment on the same prediction errors are done to improve the embedding capacity (Kamal and Mahfuzul Islam, 2015). Nevertheless, it destroys its prime objective of managing image quality. However, still embedding capacity are demanded to be improved.

Another very exclusive contribution by Ong et al. (2014) is noted in the literature which intentionally and completely destroys the visual quality of an image at the time of embedding message. As none schemes can raze the distortion, it will be more astute to destroy the image quality intentionally in a large scale. Then the introducer can be decoyed to oversight noisy image. Besides, many of the applications like forensic and medicine require having the cover information undisclosed and suitable for reconstruction (Kamal and Mahfuzul Islam, 2014), another unique contribution of the authors. To achieve these two targets, the embedding is done in (Ong et al., 2014) by Histogram Association Mapping (HAM). That HAM policy is developed to work on pixels histogram. In another scheme the capacity of that HAM methodology is improved by working on the histogram of prediction errors rather than the pixels' one (Kamal and Mahfuzul Islam, 2016). However, both the schemes suffer from imbalance partitions of gray scale and longer side information. The problems are clearly discussed in the following section. The solutions to these issues at HAM embedding are presented in that article.

The article is organized in several sections. Section II will describe the related work and some observations on it. Section III is presented to mitigate those observations. Experimental results are demonstrated at section IV. Section V is to analyze the performance statistically and section VI is to conclude the article.

\section{Related Work and Observations on it}

The article is devoted to mitigate some of our observations on the novel work of Ong et al. (2014). Therefore, in this section the work of Ong et al.
(2014) is outlined as a related work and lastly our observations are demonstrated.

The image is first divided into $N$ non-overlapping blocks. Henceforth, minimum and maximum of each block, (min, max), are identified to calculate the range's value, $R=$ max-min +1 . Blocks are classified as Reflective Block (RB) or Non-Reflective Block (NRB) settling on whether $\mathrm{R}<128$ or not. For each block, gray scale is divided into $P=\lceil 256 / R\rceil$ partitions, e.g., for $P=4$, gray partitions are (0-63), (64-127), (128-191) and (192-255). For the convenience, in all the following examples these partition ranges will be referred to point to a specific partition. Then, bins of histogram of a block should be situated to a single partition, e.g., (64-127), which is termed as 'origin partition' and the others are 'reflective', i.e., (0-63), (128-191) and (192-255). The situation is depicted in Fig. 1. During the embedment the number of bits that a block can conceive is $n=\log _{2} P$ bits, here $n$ will be 2. In HAM, reflective partition is chosen harmonizing the patterns of the embedding bits i.e., to embed $00,01,10$ or 11 the association and the translation of pixel values of the block will be made to itself, partition (0-63), (18-191) or (192-255) respectively. The green binary bits inside into the boxes in Fig. 1 represent the chunk of message bits. Based on the pattern of these bits HAM is performed. However, some observations on that novel work are identified and are presented in the following.

Observation 1: Consider that $P=4, \min =15$ and $\max =72$ of a block $B_{i}$.

Then frequency bars of the histogram will take place at partition 1 and 2 at a time which is in fact an unsolvable problem by HAM scheme of Ong et al. (2014) because original block cannot be defined here. The solution of the situation if the block resides at multiple partition is not outlined by Ong et al. (2014). The scenario is depicted in Fig. 3a.

Observation 2: If $R>127$ then number of bins in original partition is greater than reflective partition. Hence, reflection by HAM is not possible as that will engender a mapping anomaly. At embedding ' 1 ', Ong et al. (2014) performs bins' association by shifting half of the pixels by min, that is $x$-min and other half by max, which is $x+\max$. The scenario is depicted in Fig. 4a. Consider again, as the same given in the article of Ong et al. (2014) $\min =10$ and $\max =$ 252 . Then bin 250 will associate to bin $502(=250+252)$ which will undoubtedly exceed the gray range.

Observation 3: A 'side information' is managed by Ong et al. (2014) to assist the receiver in extracting embedded information. The min and the max values of each block are stored at side information during embedment process. Therefore, each block increases the side information by 2 bytes. The embedding capacity (number of bits embedded per pixel) depends on two issues-range $R$ and size of blocks. Because, the number of partitions $P$ in the gray scale as well as the 
embeddable bits to a block $n$ are reciprocal to $R$. Again smaller size of image block will increase the number of blocks in the image. Each block conceives message chunk separately. Therefore, to increase capacity, block size should be smaller. Now for block size of $2 \times 1$ of the image of size $M \times M$, the number of blocks will be $N=$ $M \times M / 2$. Then the number of side information will be $2 \times M \times M / 2=M \times M$. As each min and max value come from the gray range $0-255$, the length of side information is $M \times M$ bytes which are, indeed, the actual image size. Hence length of side information is also a headache to consider for further minimization.

Mitigations of these limitations (observation 1, 2 and 3 ) are presented at following section.

\section{Mitigations of Observations}

All the limitations stated in aforesaid section are mitigated here. At RB starting point of all partitions are shifted by $2^{a}$ where the value of $a$ comes from the relation:

$$
\underset{a \in\{i, \text { where } 0 \leq i<8\}}{\arg \max }\left(\left(\min -T_{s}\right) \geq 2^{a}\right)
$$

There $T_{s}$ is the starting point of Ong et al. (2014) $i^{\text {th }}$ partition. Therefore, the starting point of $i^{\text {th }}$ partition is $T_{s}+2^{a}$ and end point is $T_{s}+2^{a}+256 / P$. The gray scale is considered as a ring. The partition is illustrated in Fig. 2. Thus allocating bins of a block to multiple partitions at its histogram is demolished. The improvement is depicted in Fig. 3b.

Again at NRB, a wheel-scale as is shown in Fig. $4 \mathrm{~b}$ is considered in shifting values at histogram bins. The inner wheel is circulated clockwise about $d$ to decide the association of a partition in the upper wheel with the inner wheel. Then the values of the upper wheel will map to the inner wheel. Thereafter, the values of a partition in the upper wheel are replaced by the associated values in the inner wheel. The value of $d$ is set to 0 for embedding ' 0 ' while assigned a larger value, less than 255 , for embedding ' 1 '.

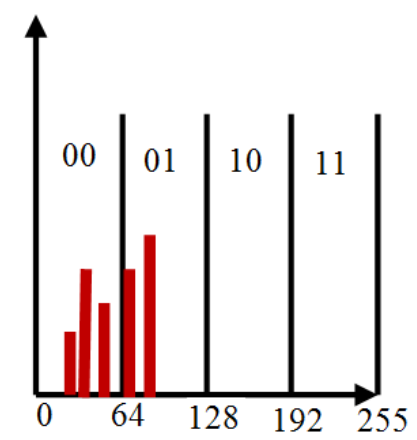

(a)
Again the side information database is minimized by storing only the min value of each block. That min value will assist the receiver to infer the original partition of cover block. At both the RB and the NRB, min value of side information will extrapolate the amount of divergence $l$ at mapping during embedment. The range $R$ will betoken to measure the number of embedded bits to the block and thus $R$ and $l$ will yield the exact embedded bits, e.g., 00, 01, 10, 11 for two bits embedment, to a block. Therefore, storing only min to side information rather than accompanying it with max value will not only minimize the length of side information by 2 but also lead the scheme to functioning itself as it should be.

Those three contributions will obviously make the Ong et al. (2014) scheme more concrete.

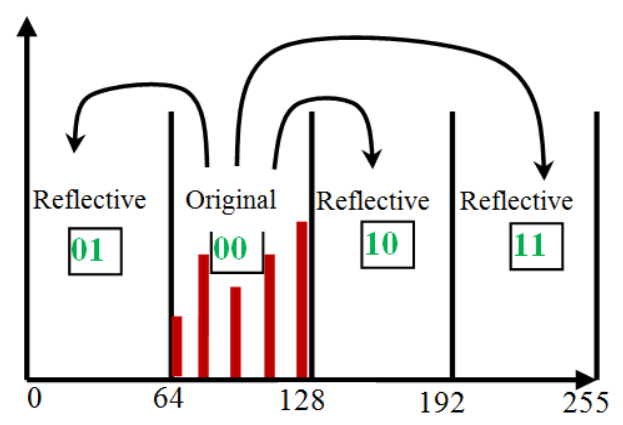

Fig. 1. Histogram association mapping

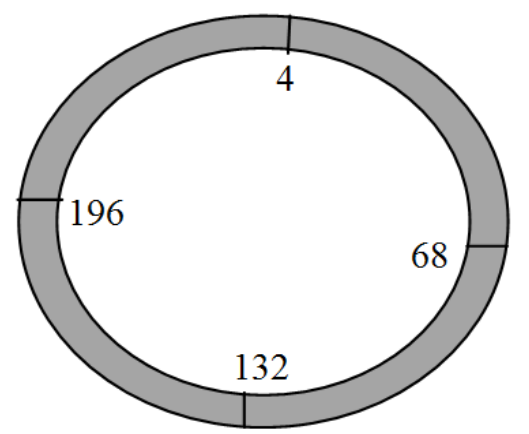

Fig. 2. Circular ribbon partition

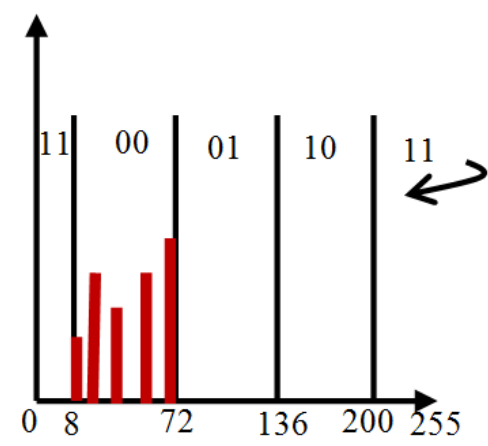

(b)

Fig. 3. Recovering partitioning problem (a) Partitions by Ong et al. (2014) (b) Proposed partitions 


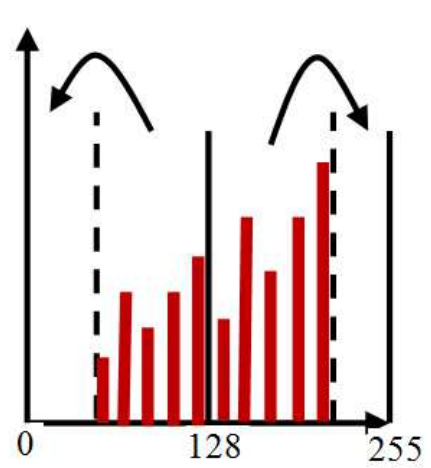

(a)

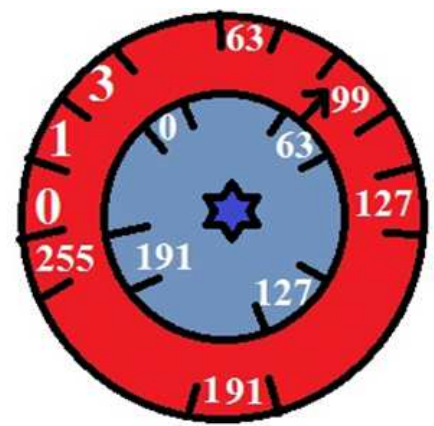

(b)

Fig. 4. Overcoming histogram shifting problem at NRB (a) Shifting by Ong et al. (2014) (b) Proposed wheel

\section{Experimental Result}

The work is experimented on more than 300 images which are either captured by our camera or collected from various research sites, online newspapers, Wikimedia, etc. As those are of different sizes the images are first resized to $360 \times 360$ pixels. For the convenient of presentations, the numerical results of only six images, of Fig. 5, are tabulated here although the proposed scheme resolved the observations in all the experimented images.

Experimentally it is observed that most of the blocks at all the images have experienced the observation 1 on Ong et al. (2014) scheme. From Fig. 6, it can be noticed that observation 1 noticeably appears at block size of 8 and 16. Thereafter, at some of the images, it sharply falls with the increment in partition range. With the increment of block size, range value $R$ will all the pixels of a block to a single partition is increased. Even then at larger block size, though the capacity will be decreased, the problem comes to be dealt noticeably whereas our proposed scheme diminishes the stated situation completely without sacrificing capacity. Proposed scheme shifted the partition to accommodate the whole block to a single partition. Thus, it nullifies the observation 1 successfully.

Another noticeable point can be defined from the Fig. 6 that Ong et al. (2014) scheme cannot embed to many blocks as these take place to two partitions in gray scale. But our proposed method has recovered the situation by shifting the partitions to adjust these to a single partition. The result is tabulated at Table 1 .

Again, at NRB the situation is opposite to RB. Figure 7 demonstrates that with the increment of block size, observation 2 swells too. This is due to having the possibility of increasing range value $R$. With the increment of block size, blocks will contain more pixels. In that case, distance of pixels of opposite border, i.e., $R$, is increased. That is the reason of increasing the frequencies of observation 2 in Fig. 7.

As the proposed scheme minimizes the stated observations in Ong et al. (2014) and it increases the embedding capacity to 6 to 17 times, noticeable at Fig. 8 and, doing so, it decreases the image quality too. The objective of the research work is to increasing embedding capacity decreasing image quality intentionally. So, proposed scheme will increase the robustness of Ong et al. (2014) from all its objectives. The result is shown in Table 2. The image distortion rate, i.e., Mean Square Errors (MSE), is measured by the following relation (2):

$$
M S E=\frac{\sum_{\substack{0 \leq i<m \\ 0 \leq j<n}}(I(i, j)-\tilde{I}(i, j))^{2}}{m^{*} n}
$$

where, I and $\tilde{I}$ represent the cover and stego image respectively and $m * n$ is the size of image.

\section{Statistical Analysis}

The objective of the statistical analysis is to realize the distortion rate of stego image. As the aim of the research work is to increase the embedding capacity resolving the anomalies found in Ong et al. (2014) scheme and to distort the visual quality of stego image in a large scale, better deviations of pixels from the originality and thus the worsening the MSE are desired. The results of MSE tabulated in Table 2 shows that the proposed scheme distorts the image more than the competing one. There are lots of other schemes in the literature those also investigate stego images statistically to realize the stego contents in an image. Those schemes usually measure the deviations of stego pixels from the cover pixels and present smaller deviations to comprehend fewer distortions, while, the proposed scheme expects to have larger distortions in the stego image. Therefore, regarding to the proposed scheme, larger values of deviations produced by those schemes are expected. Two very well known statistical analyzers, also called steganalizer and their results are explained in the following. 


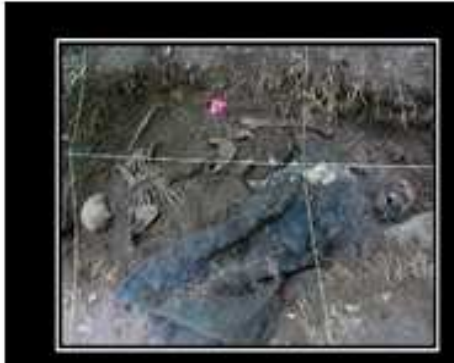

FORENSIC ANTHROPOIOGY SCHOOIS

(a)

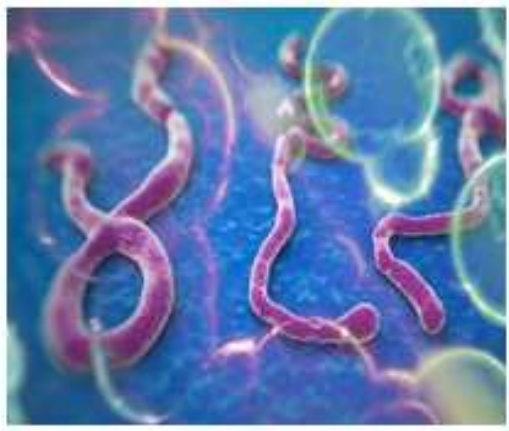

(d)

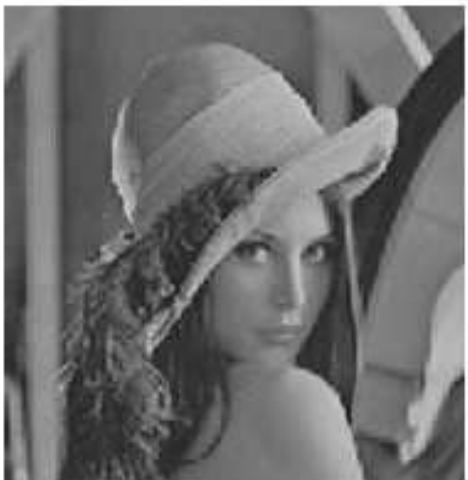

(b)

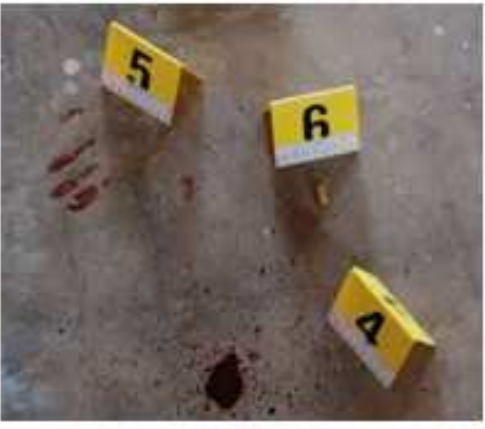

(e)

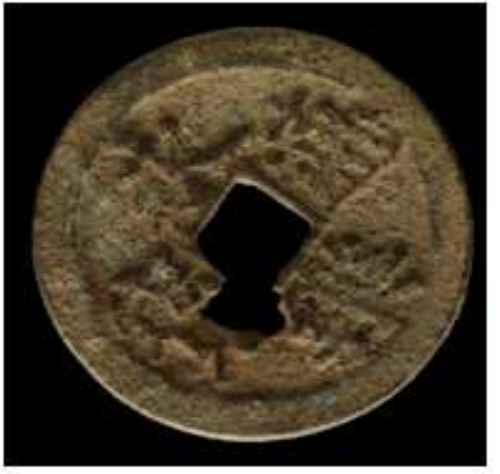

(c)

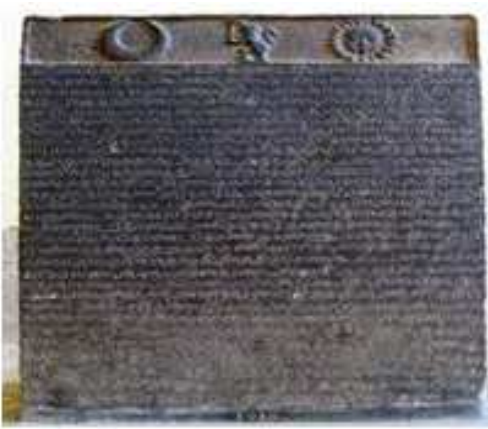

(f)

Fig. 5. Selected images from the list used in the experiment (a) Anthropology (b) Lena (c) China Coin (d) Ebola Virus (e) Blood Spot (f) Ancient Message

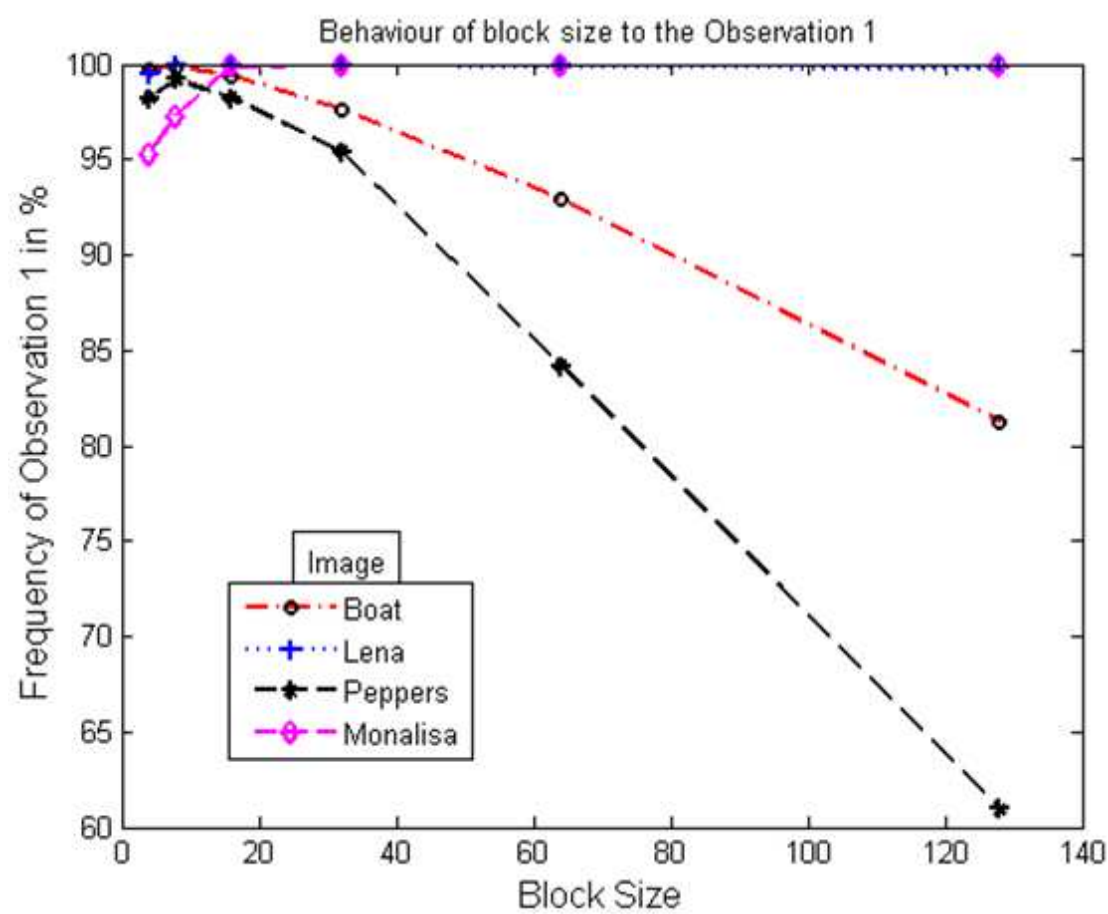

Fig. 6. Ong et al. (2014) Observation 1 on different images 


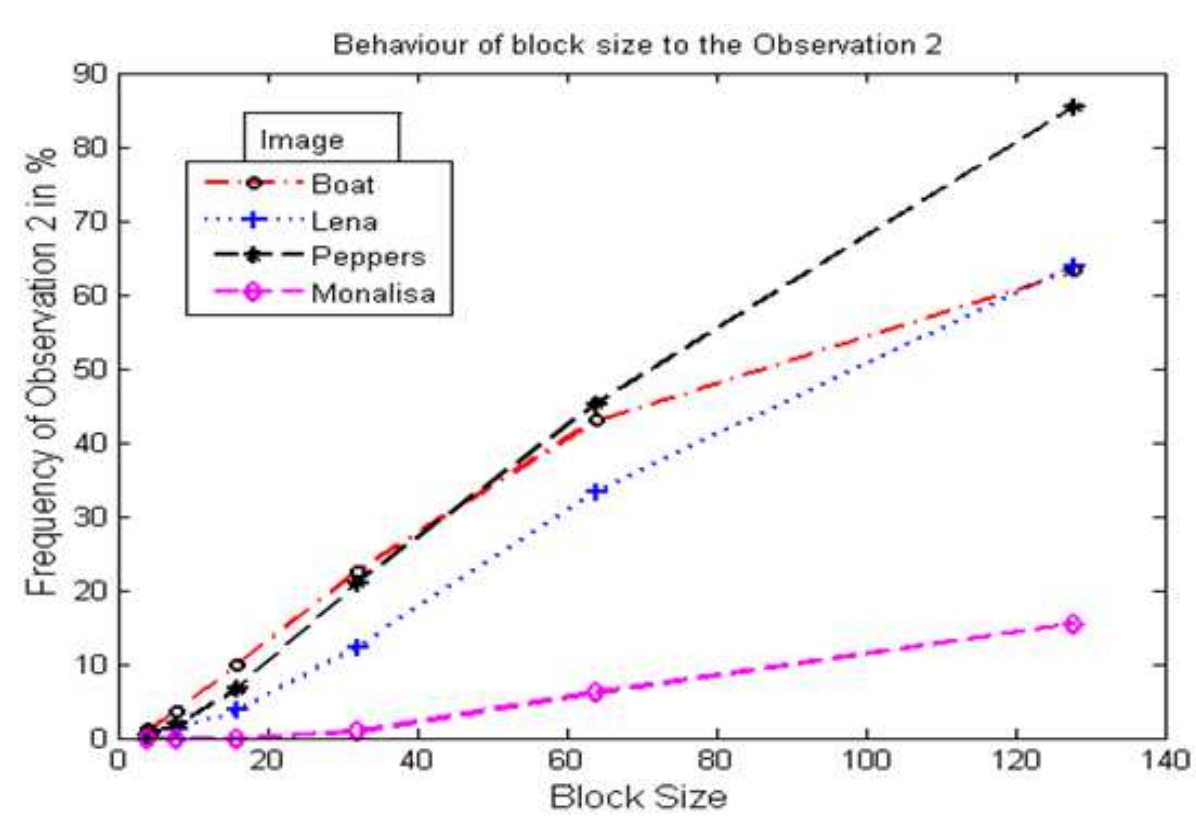

Fig. 7. Ong et al. (2014) Observation 2 on different Images

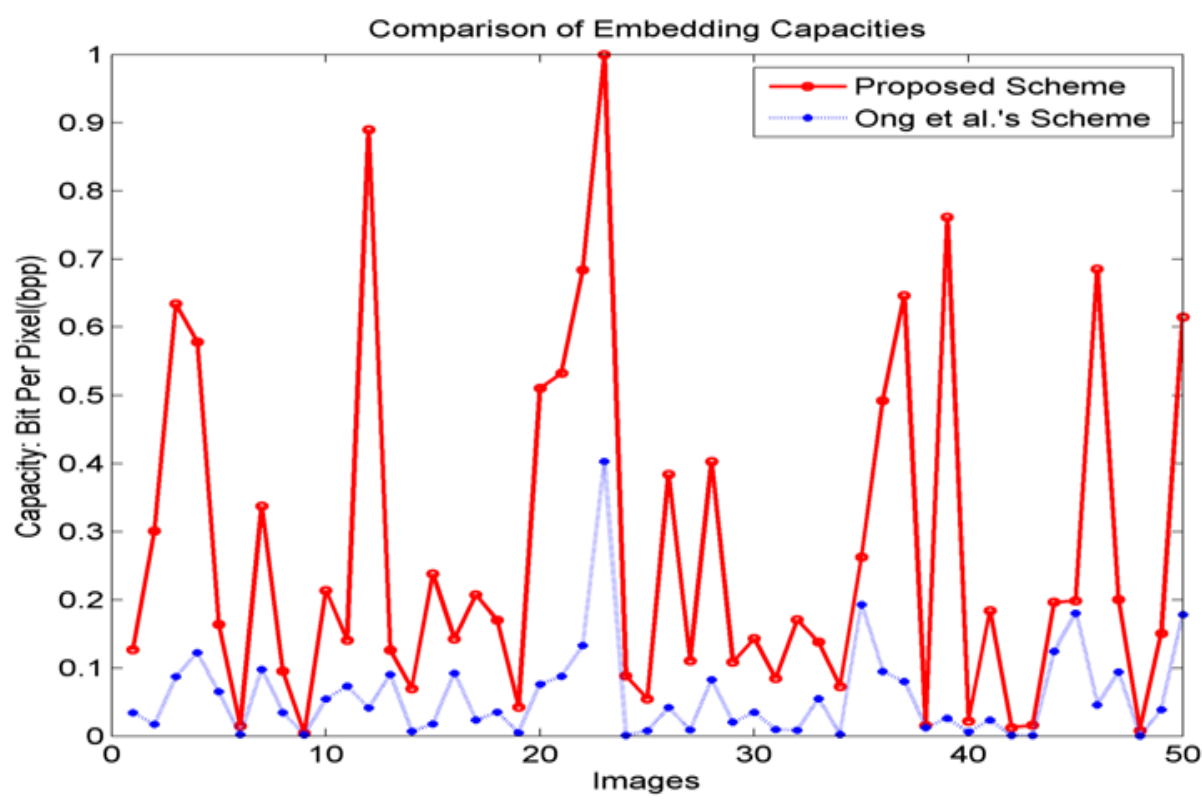

Fig. 8. Comparison of embedding capacity

Table 1. Embedding capacity at block size of 16

\begin{tabular}{|c|c|c|c|}
\hline \multirow[b]{2}{*}{ Image } & \multicolumn{2}{|l|}{ Capacity } & \multirow[b]{2}{*}{ Capacity gain" } \\
\hline & Ong et al. (2014) & Proposed & \\
\hline Anthropology & 0.02190 & 0.367300 & 16.77 \\
\hline Lena & 0.05600 & 0.890200 & 15.89 \\
\hline China coin & 0.02880 & 0.551360 & 19.14 \\
\hline Ebola virus & 0.02540 & 0.330120 & 12.99 \\
\hline Blood spot & 0.04940 & 0.456010 & 9.23 \\
\hline Ancient message & 0.04712 & 0.282410 & 5.99 \\
\hline
\end{tabular}

Capacity Gain = Capacity in the proposed/ capacity in Ong et al. (2014) scheme 
Table 2. Comparison of MSE at block size 8 and 16

\begin{tabular}{|c|c|c|c|c|}
\hline \multirow[b]{3}{*}{ Image } & \multicolumn{4}{|l|}{ MSE at } \\
\hline & \multicolumn{2}{|l|}{ Block size $=16$} & \multicolumn{2}{|l|}{ Block size $=32$} \\
\hline & Ong et al. (2014) & Proposed scheme & Ong et al. (2014) & Proposed scheme \\
\hline Boat & 0.027 & 1.476 & 0.252 & 1.580 \\
\hline Lena & 0.074 & 2.613 & 0.142 & 2.544 \\
\hline Peppers & 0.279 & 2.180 & 1.183 & 2.155 \\
\hline Mona Lisa & 0.052 & 1.519 & 0.906 & 1.636 \\
\hline Green Bangla & 0.092 & 1.411 & 1.203 & 1.834 \\
\hline
\end{tabular}

\section{Analysis using Generalized Benford's Law}

A very faster and latest technique of steganalysis is to apply Benford's law (Andriotis et al., 2013; Arshadi and Jahangir, 2014; Zaharis et al., 2011; Fu et al., 2007). A topical steganalysis based on Benford's Law, Andriotis et al. (2013). The Benford's Law states that, in a large set of natural numbers, the probability of appearing ' 1 ' as a first significant digit is higher than ' 2 ' and so on, i.e., $P(1)>P(2)>\ldots \ldots \ldots>P(9)$ where $P(n)$ is the appearing probability of $n$ in the set of natural numbers. That appearing probability of those digits, from 1 to 9 , follows a logarithmic relation:

$$
p(n)=\log _{10}\left(1+\frac{1}{n}\right), n=1, \ldots, 9
$$

$\mathrm{Fu}$ et al. (2007) again observed that Discrete Cosine Transform (DCT) coefficients do not strictly follow the rules of Equation 3. Thereafter they adapted the relation by the following equation:

$$
p(n)=N \cdot \log _{10}\left(1+\frac{1}{s+n^{q}}\right), n=1,2, \ldots, 9
$$

where, $N, s$ and $q$ defines the accuracy of the distribution sat different quality compression factors. That Equation 4 is known as generalized Benford's Law (GBL).

Before testing the stego image by the GBL, the RGB color space of the image is first converted to ycbcr color model. Thereafter, the image is partitioned into blocks of size $8 \times 8$. For every block, DCT coefficients are generated and next quantized. Those DCT coefficients are applied in GBL. The GBL first measures the total occurrences of $d$ as a first significant digit for $d=1,2, \ldots$ $\ldots$, 9. Consider totAppear $(d)$ to store the total appearances of the digit $d$ in the given set of data. Next the mean distributions of each digit are measured by:

$$
m(d)=\frac{\text { totAppears }(d)}{\sum_{i=1}^{9} \text { totAppears }(i)}
$$

A deviation between expected values, computed by Equation 4 and the mean distributions, from Equation 5, are computed for cover and stego images separately. The differences between those two deviations for each digit are used to measure the possibility of containing embedded message in the stego image. Generally, it can be expected that, for images without any or fewer embedment, the values measured by Equation 4 and 5 will be very alike and thus the measured differences of those deviations will be much closer to zero. The results of GBL for first four digits are depicted in Fig. 9 where $x$-axis and yaxis represent images and the 'difference of deviations' respectively. The figure demonstrates that the 'difference of deviations' in the proposed scheme is much larger than Ong et al. (2014) scheme in about all the images for all the digits. That higher value of 'difference of deviations' of each image implies that the distortion rate by the proposed scheme is higher and thus it dominates the Ong et al. (2014) scheme.

\section{Analysis using Subtractive Adjacency Pixels' Differences Histogram}

At natural images it is observed that histogram of vertical differences of adjacency pixels $\tilde{H}_{v}$ and horizontal differences of adjacency pixels $\tilde{H}_{h}$ stays very close to each other. Such claima are demonstrated in Zhao et al. (2009) and Hong et al. (2012). Hence, it can be anticipated that square root of summation of squared of differences of corresponding bins in those histograms should be very closer to zero. Therefore, square root of summed squared differences up to a threshold $T$ will also be small and can be calculated by the following Equation 6:

$D=\left(\sum_{i=-T}^{T}\left(\left(\breve{H}_{h}(i)-\breve{H}_{v}(i)\right)^{2}\right)^{1 / 2}\right.$

For the entire test images, setting $T$ to 40 , proposed method figures lower value of $D$ than Ong et al. (2014) scheme. 
Difference of deviations for digit 1

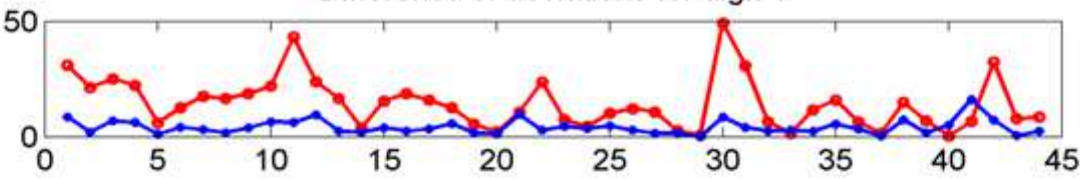

Difference of deviations for digit 2

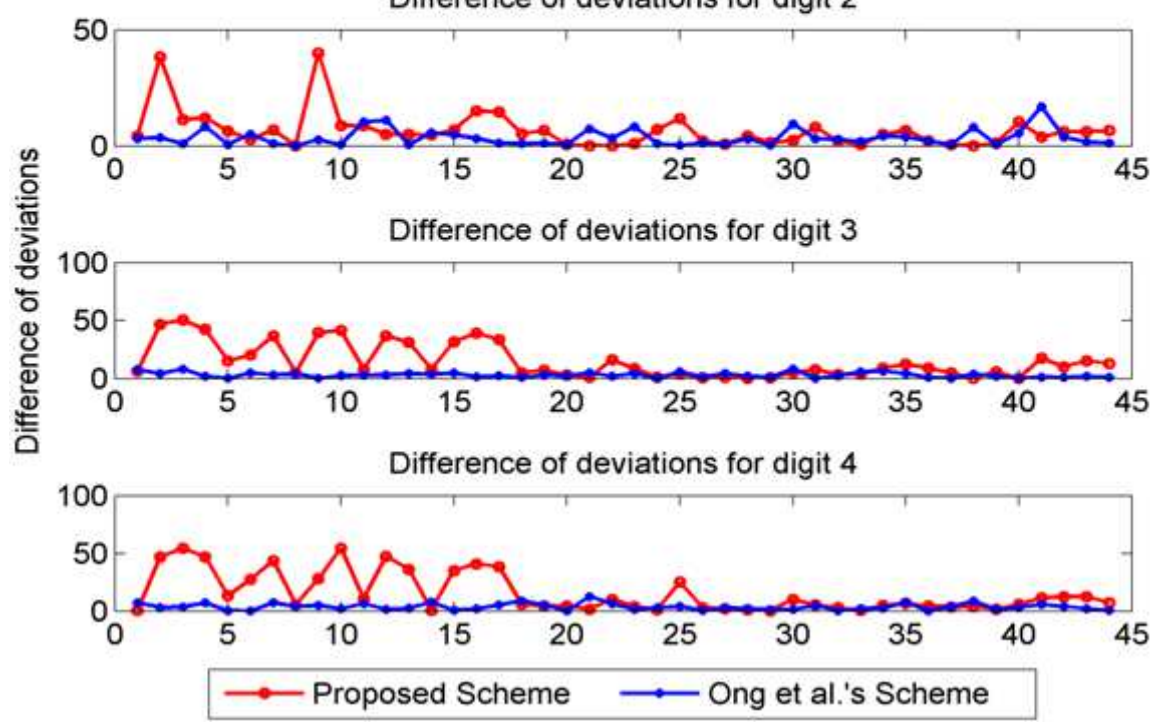

Fig 9. Analysis of digit using generalized Benford's Law

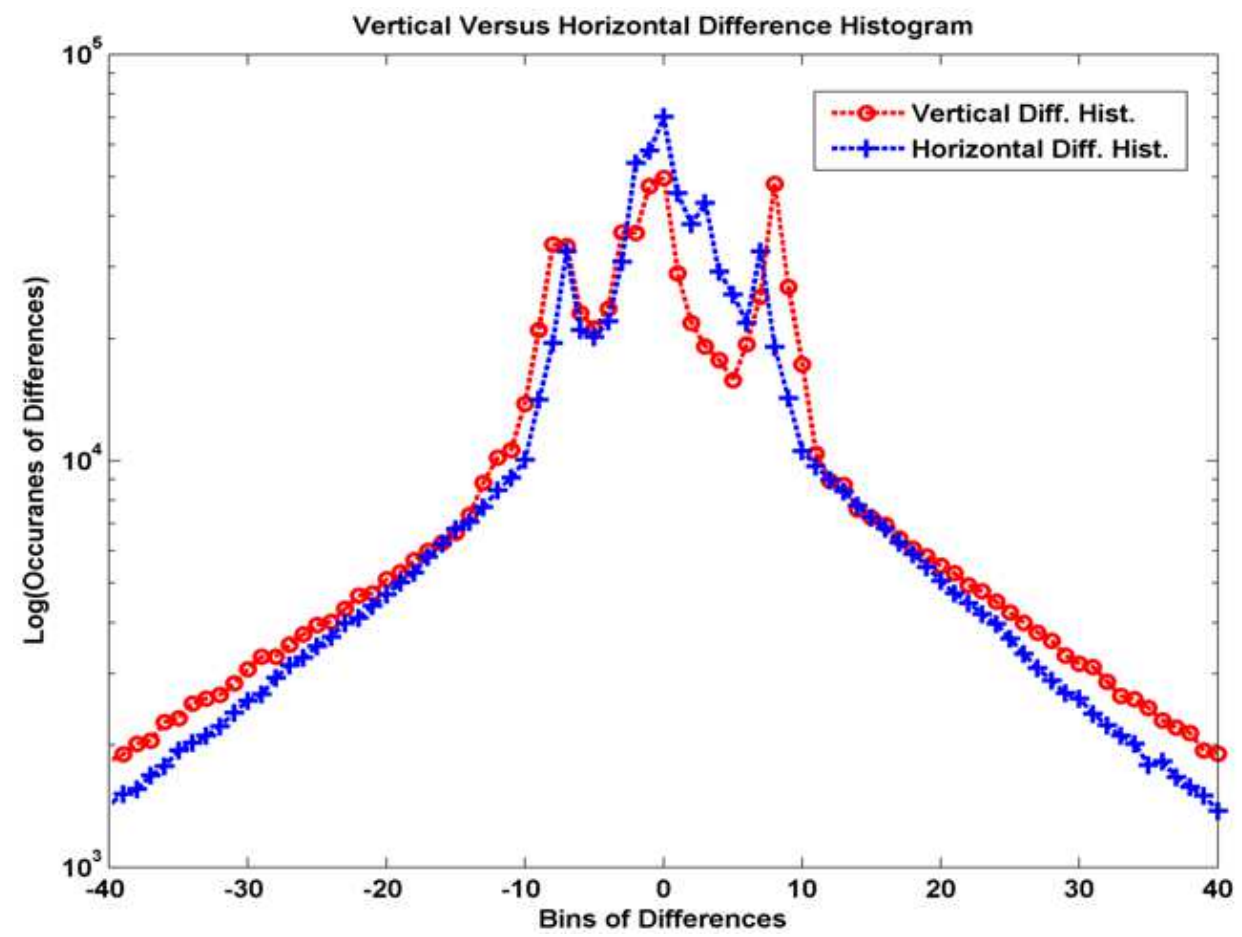

Fig. 10. Vertical versus horizontal difference histograms

From Fig. 10 it can be observed that vertical difference histogram and horizontal difference histogram present unlike results. According to the objective of the proposed work, it is also desirable. 


\section{Conclusion}

Intentionally distorting images at embedding time is a very rear attempt in image steganography. From that sense Ong et al. (2014) scheme is a very exceptional one. However, some observations on it come to our notice. These limitations can make that novel scheme useless because the stated observations are noticed in the experimental results very frequently. That is, these observations play important roles against the functionality of the embedding procedure. Alleviation of these limitations by that proposed scheme will obviously increase the robustness of Ong et al. (2014) procedure. Hence, certainly, contribution of the proposed scheme will be a notable one in the field of the image steganography. In our future works we hope to boost the embedding capacity employing prediction error schemes.

\section{Acknowledgment}

Two fellows, A.H.M. Kamal and Habiba Sultana, of ICT division of Ministry of Post, Telecommunication and Information Technology of Bangladesh are working with that research jointly. Therefore, authors are grateful to that Ministry of Bangladesh.

\section{Author's Contributions}

Habiba Sultana was working under the supervision of A.H.M. Kamal during her master program. Again A.H.M. Kamal is working as a $\mathrm{PhD}$ student under the supervision of Mohammad Mahfuzul Islam. Under the guidance of A.H.M. Kamal the structural and mathematical model was developed by Habiba Sultana. She also participated to the setup of experiment. By the direction of Mohammad Mahfuzul Islam, the problems are experimented and finally the article is written by A.H.M. Kamal.

\section{Ethics}

The article is read by all the authors. All the authors have significant contributions to the development of the contents of the paper. The work is not submitted or published elsewhere.

\section{References}

Andriotis, P., G. Oikonomou and T. Tryfonas, 2013. JPEG steganography detection with Benford's Law. Digital Investigat., 9: 246-257.

DOI: 10.1016/j.diin.2013.01.005

Arshadi, L. and A.H. Jahangir, 2014. Benford's law behavior of Internet traffic. J. Netw. Comput. Applic., 40: 194-205.

DOI: $10.1016 /$ j.jnca.2013.09.007
Chen, X., X. Sun, H. Sun, Z. Zhou and J. Zhang, 2013. Reversible watermarking method based on asymmetric-histogram shifting of prediction errors. J. Syst. Software, 86: 2620-2626. DOI: $10.1016 /$ j.jss.2013.04.086

Fu, D., Y.Q. Shi and W. Su, 2007. A generalized Benford's law for JPEG coefficients and its applications in image forensics. International Society for Optics and Photonics.

Hong, W. and T.S. Chen, 2010. A local variancecontrolled reversible data hiding method using prediction and histogram-shifting. J. Syst. Software, 83: 2653-2663. DOI: 10.1016/j.jss.2010.08.047

Hong, W., 2012. Adaptive reversible data hiding method based on error energy control and histogram shifting. Opt. Commun., 285: 101-108. DOI: 10.1016/j.optcom.2011.09.005

Hong, W., T.S. Chen and C.W. Luo, 2012. Data embedding using pixel value differencing and diamond encoding with multiple-base notational system. J. Syst. Software, 85: 1166-1175. DOI: $10.1016 /$ j.jss.2011.12.045

Kamal, A.H.M. and M. Mahfuzul Islam, 2014. Facilitating and securing offline e-medicine service through image steganography. Healthcare Technol. Lett., 1: 74-79. DOI: 10.1049/htl.2013.0026

Kamal, A.H.M. and M. Mahfuzul Islam, 2015. Capacity improvement of reversible data hiding scheme through better prediction and double cycle embedding process. IEEE International Conference on Advanced Networks and Telecommuncations Systems, Dec. 15-18, IEEE Xplore Press, Kolkata, pp: 1-6. DOI: 10.1109/ANTS.2015.7413636

Kamal, A.H.M. and M. Mahfuzul Islam, 2016. Enhancing the embedding payload by handling the affair of association and mapping of block pixels through prediction errors histogram. Proceedings of the International Conference on Networking Systems and Security, Jan. 7-9, IEEE Xplore Press, Dhaka, pp: 1-8. DOI: 10.1109/NSysS.2016.7400691

Lu, Y.Y. and H.C. Huang, 2014. Adaptive reversible data hiding with pyramidal structure. Vietnam J. Comput. Sci., 1: 1-13. DOI: $10.1007 /$ s40595-014-0020-y

Ni, Z., Y.Q. Shi, N. Ansari and W. Su, 2006. Reversible data hiding. IEEE Trans. Circuits Syst. Video Technol., 16: 354-361. DOI: $10.1109 /$ TCSVT.2006.869964

Ong, S.Y., K.S. Wong and K. Tanaka, 2014. A scalable reversible data embedding method with progressive quality degradation functionality. Signal Process.: Image Commun., 29: 135-149. DOI: 10.1016/j.image.2013.09.001 
Tai, W.L., C.M. Yeh and C.C. Chang, 2009. Reversible data hiding based on histogram modification of pixel differences. IEEE Trans. Circuits Syst. Video Technol., 19: 906-910.

DOI: 10.1109/TCSVT.2009.2017409

Tsai, P.Y., Y.C. Hu and H.L. Yeh, 2009. Reversible image hiding scheme using predictive coding and histogram shifting. Signal Process. 89: 1129-1143. DOI: $10.1016 /$ j.sigpro.2008.12.017
Zaharis, A., A. Martini, T. Tryfonas, C. Ilioudis and G. Pangalos, 2011. Lightweight steganalysis based on image reconstruction and lead digit distribution analysis. Int. J. Digital Crime Forens., 3: 29-41. DOI: $10.4018 /$ jdcf.2011100103

Zhao, H., H. Wang and M.K. Khan, 2011. Statistical analysis of several reversible data hiding algorithms. Multimedia Tools Applic., 52: 277-290.

DOI: $10.1007 / \mathrm{s} 11042-009-0380-\mathrm{y}$ 